\title{
Origin of Nuclear Mitochondrial Pseudogenes (Numts)
}

\section{Nasser-eddin Rateb Dweik}

Department of Agriculture, Al- Quds Open University, Jericho, Palestine

*Corresponding author: Nasser-eddin Rateb Dweik, Department of Agriculture, Al- Quds Open University, 1804 Jericho, Palestine, Tel: 00972 568147021; E-mail: dweik500@gmail.com

Receiving date: Sep 26, 2017; Acceptance date: Nov 06, 2017; Publication date: Nov 09, 2017

Copyright: $\odot 2017$ Dweik NR. This is an open-access article distributed under the terms of the Creative Commons Attribution License, which permits unrestricted use, distribution and reproduction in any medium, provided the original author and source are credited.

\begin{abstract}
Mitochondria are well-defined cytoplasmic organelles of eukaryotic cells, which take part in various cellular metabolic functions, mainly cellular respiration. Several researchers have tried to validate the mechanism of gene transfer between the mitochondria and the cell nucleus. However, some scientific facts show it is very difficult or even impossible for a researcher to confirm DNA transfer process from a living organelle, because mitochondria possess certain specific features that prevent certainty of the DNA transfer process: Such as; dynamicity of mitochondrial organelles, exist in big numbers, can change its location, size and shape, however, gene transfer techniques lack precision. Healthy active mitochondrion has perfect double membranes that sustain its function of keeping in all its constituents. So, most probably the pseudogenes or Numts that are detected in eukaryotic nuclei do not come from living mitochondria rather from aged degraded ones. However, it may come from misplaced DNA segments resulted from any gene transfer process.
\end{abstract}

Keywords: Nuclear Mitochondrial Pseudogenes (Numts); Mitochondrial gene transfer; Endosymbiosis

\section{Introduction}

Eukaryotic cells compartmentalize their biochemical processes within organelles, which have specific functions that must be maintained for overall cellular health. Several researchers have tried to confirm reality of gene transfer between mitochondria and a cell nucleus using mainly yeast cells. This process was assumed to have happened more than one billion years ago between a guest prokaryotic organism and a host eukaryotic cell, where the prokaryotic organism abandoned most of its unnecessary genes and became an organelle inside a eukaryotic cell. This proposed relationship is called Endosymbiosis.

Certain similarities have been detected among some mitochondrial sequences and nuclear nonfunctional sequences. These nonfunctional sequences that encountered in eukaryotic nuclei are called Nuclear mitochondrial pseudogenes (Numts). Scientists have detected at least 13 Numts in the chicken nuclear genome. Identities between Numts and mitochondrial sequences varied from 58.6 to $88.8 \%$ [1]. In most cases, transposed mitochondrial sequences lack functional promoters and regulatory elements, and therefore they contribute to the accumulation of mutations.

In reality, mitochondria are very dynamic organelles, capable of changing size and shape in a matter of seconds [2]. Observations of living cells cultured in media have shown mitochondria to be dynamic organelles that change their shape, move from place to place within the cytoplasm, and undergo both fusion and fission [3].

The chondriome of Saccharomyces cerevisiae is typically organized as a network of interconnected tubules. This yeast dramatically regulates the shape, size, and number of its mitochondria during cell growth [3]. It was found that the number of mitochondria increases when cells of Saccharomyces cerevisiae are under glucose-limiting conditions [4].
Mitochondria cannot be created de novo, means that any new mitochondrion must be formed from the division of an existing organelle [2]. The number of mitochondrial units in a particular cell type is determined by its energetic demand. Through division and fusion processes, mitochondria enable an effective adaptation of the mitochondrial organelle to the metabolic needs of the cell [5].

The number of virtual mitochondria per cell appears to be closely regulated within a given cell type but differs widely from cell type to another. For example, in mammalian liver cells the number of mitochondria varies from 1000-2000 per cell [6], while the average number in Saccharomyces cerevisiae was revealed to be 22 mitochondrion per cell [7].

\section{Main text}

Let's agree to certain aspects before commenting on reality of the proposed gene transfer mechanism like, source and reality of such mechanism and the DNA empty mitochondria. First of all, where did mitochondria get the gene transfer mechanism? Neither prokaryotic nor eukaryotic organisms would do such a suicidal process. However, red blood cells function is maintained after getting rid of its nuclei and not parts of its genome. Nevertheless, DNA empty mitochondria that have been detected in some organisms do not necessarily mean they have lost all their genes; however, it may not have DNA from the beginning, where all DNA copies have migrated to one side of a dividing cell and left the other side empty.

During the isolation process in the Lab, quite a lot of mitochondria will be lost as they tend to burst out during centrifugation steps. When some mitochondria burst they cause a DNA mix-up. So, researcher cannot differentiate between mitochondrial DNA and any DNA entangled or misplaced in the cytoplasm. Even after isolation, mitochondria do not stay healthy for longer than a few hours. On the other side, mitochondria respond very fast to stress. The stress given by isolation method would produce changes in mitochondria including increase in fission. 
Several researchers tried to validate the Endosymbiosis mechanism of gene transfer process from the mitochondrion (or a prokaryote) to the cell nucleus of a eukaryotic organism. The Endosymbiosis hypothesis implies that a cooperative relationship has been established between the prokaryotic organism and a host organism (eukaryote), where the prokaryotic organism abandoned its unnecessary functions and became an organelle. However, up to my knowledge, no one has sequenced several mitochondrial chromosomes of an individual organism and found them variable in length, i.e, have lost different variable DNA segments.

As a matter of fact, mitochondria do senesce and like most cellular components, they are recycled on a regular basis [8]. Cells may break down mitochondria when they are not working properly or when it ages [9]. A lot of mitochondrial proteins are encoded in the nucleus. Critical mutations in one of the genes that are essential for respiratory function can impair respiration and cause mitochondria stop working.

However, mitochondria have a couple of ways of generating ATP. The oxidative phosphorylation method generates some amount of free radicals as a natural byproduct. Most likely, free radicals damage mitochondria causing release of components. A growing body of evidence has established that the levels of Reactive Oxygen Species and oxidative damage to lipids, proteins, and nucleic acids are significantly increased with age in animal and human tissues [9].

On the other side, Scientists have used high-velocity microprojectile bombardment method successfully to transfer genes into organelles. In this method, usually enormous numbers of copies of the desired (foreign) DNA segments are placed onto the tungsten particles. When using the microprojectile method it is not easy to determine the destiny of DNA segments. As a matter of fact, bombarded DNA segments will not enter each organelle, some organelles may receive a DNA segment while others may not, some other DNA segments will be placed on the surface of organelles or deposited in the cytoplasm. Some of those segments that are placed on the surface of organelles or deposited into cytoplasm may migrate and enter the cell nucleus (assuming the organism's nucleus is kept away from targeted organelles). It is really difficult or even impossible to determine the destination of bombarded DNA segments, because of mitochondrial dynamicity.

During degradation of any mitochondrion a mitochondrial DNA segment may migrate and inter the nucleus, integrate with any nuclear chromosome and become a pseudogene. So, most probably transposed mitochondrial DNA does not come from a living organelle but from dead degraded one or from entangled or misplaced DNA segments resulted from any gene transfer process.

As mentioned before, scientists have detected at least 13 Numts in the chicken nuclear genome [1]. Although, the identities between Numts and mitochondrial sequences are not high enough (58.6 to $88.8 \%$ ), however, it is considered a real transposition of mitochondrial DNA to the nucleus. However, such result refers to the following:

The number of transposed sequences is very low; 13 in about 150 million years of the proposed chicken life on earth, so, it is a very lowincidence process.

Depending on the previous point, how is it possible that researchers have detected transposition of their introduced genes within days or weeks? This means that what they have detected comes from contamination or from DNA segments that are misplaced in the cytoplasm.

Genetics proposes, whether the transposed mitochondrial "genes" have functional promoters or promoter less it will not be recognized by eukaryotic RNA polymerase II, because it has different transcription factors and different binding sites on the DNA. Sigma factors are the transcription factors of prokaryotes, while the general transcription factors for RNA polymerase II include TFIID, TFIIA, TFIIB, TFIIF, TFIIE, and TFIIH. The binding sites of a typical promoter of prokaryotic genes are formed from hexamer bases at -35 and hexamer bases on -10 site, while a binding site of a typical promoter of eukaryotic genes is formed from hexamer bases at -25 . Nevertheless, foreign genes have not regulatory elements to activate them.

The source of the transposed mitochondrial DNA (Numts) could come from dead or degrading organelles and not from living ones. Integrating a DNA segment from a dead degrading organelle does not validate the Endosymbiosis mechanism of gene transfer.

Nevertheless, whether scientists have implemented the microprojectile method in vivo or on isolated mitochondria, or if the targeted mitochondria belong to a wild- strain or it belongs to a mutant strain that does not contain any DNA; like rho0 strain [10], researchers have to answer important questions before they announce their results.

The presence of many organelles in each cell imposes a big obstacle in the way of Endosymbiosis gene transfer mechanism. After application of any mitochondrial gene transfer technique; which organelle will start sending part of its DNA to the nucleus? It is unlikely that most organelles will start sending parts of their DNA to the nucleus because they will lose their genetic material and ruin their function. For example, losing one or two of the mitochondrial transfer RNA genes will hinder the translation process and subsequently hinder protein synthesis, which is detrimental to energy production process. Most likely only those who received the foreign DNA will get rid of the new visitors. Such process means that the mitochondrial organelles are programmed at certain rules and disciplines; where they reject foreign genes only and keep original ones. So, this process does not validate the Endosymbiosis gene transfer mechanism.

\section{DNA detection}

As a matter of fact, researchers cannot distinguish between organelles that have received a desired DNA segment (or a plasmid) and those they have not? When a researcher wants to check if his targeted organelles have acquired (transformed) his desired gene. Presumably, he picks a certain organelle, crushes it, and then he takes its DNA out for detection. Upon doing so, if he found his desired gene in a certain organelle, how could he be certain that this organelle will send its foreign DNA to the nucleus in the near future? On the other side, if he does not find his desired gene in a certain organelle, how could he be certain that this organelle has acquired or possessed (get integrated) his desired gene first and then sent it to the nucleus? However, it may not acquire the desired gene from the beginning [11].

Someone may say, losing one or two of the mitochondrial genes will not hinder protein synthesis because each mitochondrion has several copies of the mitochondrial DNA chromosome, if one gene is lost, the other copy will compensate for its protein. This is true if the DNA shearing process is truly exist, it occurs at long intervals and the 
process can drive out intact promoter-modified genes, however, researchers have to answer the following questions:

First of all, where does the DNA transfer mechanism come from [12]? Organisms struggle to stay alive and they do not do such a suicidal process (assuming a mitochondrion was a prokaryote).

What is the mechanism behind the DNA shearing process? Is it done by restriction enzymes or by other mechanism? How can the cutting point at the DNA level be determined in order to drive out intact genes? However, transposed intact genes cannot be expressed in the nucleus unless their promoters are modified to resemble eukaryotic ones [12].

In the proposed Precambrian era, how the first mitochondrion managed with one chromosome copy or it was having several copies from the very beginning? However, what would the loss of several mitochondrial genes through a haphazard mechanism do to an organism as long as nuclear- integrated intact genes would become pseudogenes? It is known that the eukaryotic transcription apparatus cannot deal with prokaryotic genes unless their promoters are modified. However, why isn't this destructive process terminated or cancelled by natural selection?

Upon gene tracking in the cell nucleus; how can researchers be certain that the tracked gene comes from an intact organelle, degraded organelle or from the cytoplasm? If researchers could not determine the source of the tracked gene then we most probably assume it comes from the cytoplasm (degraded organelle or misplaced one) and not from alive mitochondria.

On the other side, If a nuclear gene (does not have a function in the organelle) is injected directly into a nucleus, and then detected in an organelle. Researchers have to answer how it enters the organelle. Organelles are not open to any DNA segment. It is known that organelles have receptors on their membranes for signal peptides (polypeptides) and not for DNA sequences. If a researcher has detected a nuclear gene in an organelle, then there must be a DNA contamination or a mistake somewhere in the DNA isolation process. Moreover, if microinjection becomes a practical method for delivering DNA segments directly into mitochondria, again researchers still cannot be certain which one is injected because, mitochondria are very dynamic organelles, capable of changing location, size and shape. If later detected in the nucleus, then we have to think of probable contamination or a mistake somewhere.

\section{Conclusion}

It is difficult or even impossible for a researcher to determine the source of his detected foreign genes; whether they have migrated to the nucleus from healthy active mitochondrion or from DNA segments found in the cytoplasm. As long as researchers cannot be certain about the source of their tracked genes, then they cannot validate the Endosymbiosis gene transfer mechanism. Finally, editors have to be very careful upon accepting such articles, and published articles have to be revised and to take into consideration all mentioned remarks.

\section{References}

1. Pereira SL, Baker AJ (2004) Low number of mitochondrial pseudogenes in the chicken (Gallus gallus) nuclear genome: implications for molecular inference of population history and phylogenetics. BMC Evol Biol 4: 17.

2. Kempken F (2011) Plant Mitochondria, Advances in Plant Biology. Springer Science+ Business Media, LLC, NY 10013, USA.

3. Hermann GJ, Shaw JM (1998) Mitochondrial dynamics in yeast. Annu Rev Cell Dev Biol 14: 265-303.

4. Visser W, van Spronsen EA, Nanninga N, Pronk JT, Kuenen JG (1995). Effects of growth conditions on mitochondrial morphology in Saccharomyces cerevisiae. Antonie Van Leeuwenhoek 67: 243-253.

5. Braun RJ, Westermann B (2011) Mitochondrial dynamics in yeast cell death and aging. Biochem Soc Trans 39: 1520-1526.

6. Robin ED, Wong R (1988) Mitochondrial DNA molecules and virtual number of mitochondria per cell in mammalian cells. J Cellular Physiol 136: 507-513.

7. Lewin B (2004) Genes VIII. Pearson Prentice Hall, NJ.

8. Campbell CL, Thorsness PE (1998) Escape of mitochondrial DNA to the nucleus in ymel yeast is mediated by vacuolar-dependent turnover of abnormal mitochondrial compartments. J Cell Sci 111: 2455-2464.

9. Wei YH, Lu CY, Lee HC, Pang CY, Ma YS (1998) Oxidative damage and mutation to mitochondrial DNA and age-dependent decline of mitochondrial respiratory function. Ann N Y Acad Sci 854: 155- 170.

10. Fox TD, Sanford JC, McMullin TW (1988) Plasmids can stably transform yeast mitochondria lacking endogenous mtDNA. Proc Natl Acad Sci USA 85: 7288-7292.

11. Karp G (2002) Cell and Molecular Biology. 3rd edition, John Wiley and sons, New York, USA.

12. Dweik NR (2016) Experimental Sciences and Endosymbiosis Hypothesis: Solving a Paradox. J Nat Sci Res 6: 124-130. 